\title{
FAR INFRARED EMISSION FROM AN INTERGALACTIC DUST CLOUD?
}

\author{
Bogdan Wszolek and Konrad Rudnicki \\ Jagellonian University Observatory \\ Cracow, Poland
}

\author{
Sivia Masi and Paolo de Bernardis \\ Department of Physics \\ University "La Sapienza" \\ Rome, Italy
}

The possible presence of general extragalactic extinction has been studied by many authors. Extragalactic dust can be associated with a pregalactic star population and/or it can be ejected by galaxies during their evolution.

Four individual extragalactic dust clouds have been described in the literature (Rudnicki and Wszolek 1986) using several statistical techniques, all involving the degradation of the optical radiation coming from distant galaxies. The presence of the Abadi and Edmunds Cloud has also been detected using H I $21 \mathrm{~cm}$ measurements, while for the other clouds, either similar detections or comparison with existing data were not published till now or they have not given positive results. Far-infrared (far-IR) emission is expected from these dust clouds because their temperature must be greater than the cosmic microwave background temperature; however, the grain composition and the physical conditions of the extragalactic dust are completely unknown. For this reason the spectrum of such emission cannot be predicted quantitatively.

The IRAS satellite has provided an almost complete sky coverage in four IR wavelength bands $(12,25,60$, and $100 \mu \mathrm{m})$. The sensitivity to diffuse radiation was high enough to detect emission from galactic dust clouds with temperatures of $\sim 20 \mathrm{~K}$ and optical extinction much lower than $1^{\mathrm{m}}$; one can expect that extragalactic clouds with similar absorption and temperature values could be found in the IRAS data set.

We began this investigation by studying the Okroy (1965) Cloud, which lies at high galactic latitude $\left(l=304^{\circ} .4, b=82^{\circ} .6\right.$ ) where the local background is very low (about $10 \mathrm{MJy}$ $\mathrm{sr}^{-1}$ at $100 \mu \mathrm{m}$ ). The absence of galaxies in this region is easily visible in the Zwicky catalog as can be seen readily in the map reported by de Lapparent, Geller, and Huchra (1986).

Murawski (1983) performed a comprehensive study of the number of galaxies as a function of magnitude as well as of the mean colors of galaxies in the Okroy cloud area and in a reference area. Such a comparison proved that there is a deficiency of galaxies in the Okroy cloud area because of an extinguishing cloud. He found a mean extinction equal to $0{ }^{m} 45 \pm$ 0 . 05 in blue. Using data for RR Lyrae stars, he proved that this cloud lies behind the spherical star halo of our galaxy, i.e., that it is an extragalactic cloud.

A map of $100 \mu \mathrm{m}$ emission in this region is shown in Figure 1. This has been constructed using the first IRAS sky coverage. The analysis of the second coverage gives similar results, thus excluding spurious effects like striping or detector noise. A large-scale galactic and ecliptic gradient of emission is evident in the map; also a local emission enhancement is visible in a region a few degrees wide, centered at R.A. $=195^{\circ}, \delta=20.5^{\circ}$, and elongated in the R.A. direction. The large-scale trend of the data can be removed by fitting with a smoothing function. The elongated shape is in rough agreement with Okroy's original observations.

A Gaussian profile fitting shows that the cloud emission is about $(1.3 \pm 0.1) \mathrm{MJy} \mathrm{sr}^{-1}$ at $100 \mu \mathrm{m}$ while it is lower than $0.12 \mathrm{MJy} \mathrm{sr}^{-1}$ at $60 \mu \mathrm{m}$; the dust temperature in the cloud is less than $21 \mathrm{~K}$. 


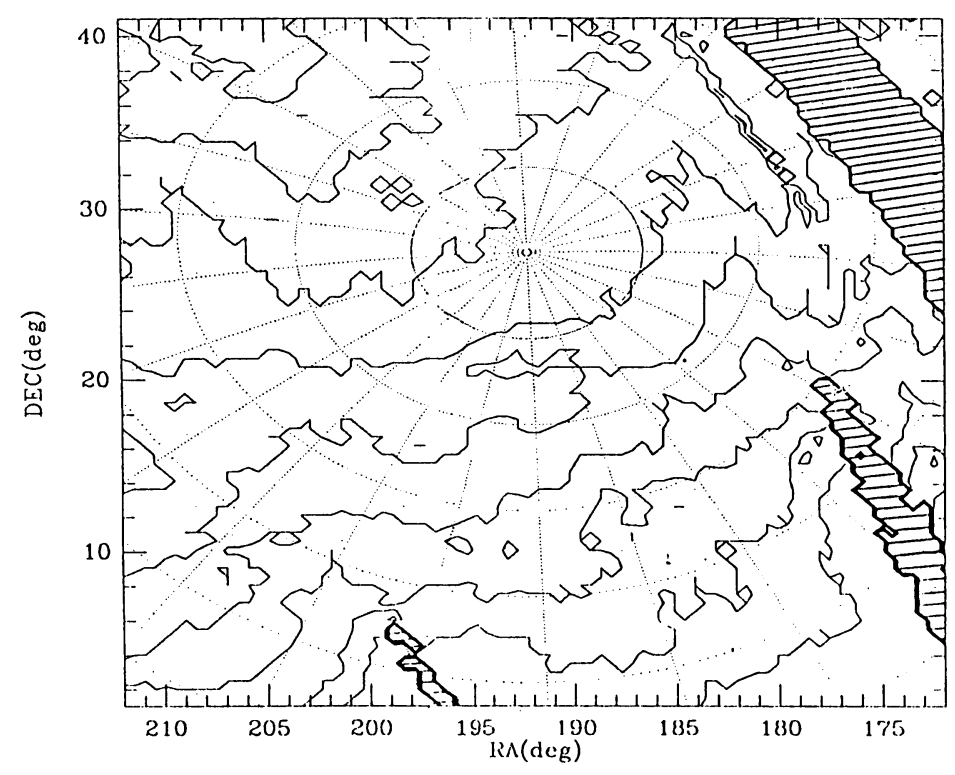

Figure 1. $100 \mu \mathrm{m}$ emission detected by IRAS in the Okroy cloud region. The isophotes have been drawn at levels between 7 and $12.5 \mathrm{MJy} \mathrm{sr}^{-1}$, with $0.5 \mathrm{MJy} \mathrm{sr}^{-1}$ increments. The Okroy cloud is evident in the center of the figure.

We conclude that the excess emission found in IRAS $100 \mu \mathrm{m}$ data in the Okroy region can be due to the presence of an extragalactic dust cloud. One cannot exclude the presence of an "IR cirrus" (Hauser et al 1984; Laurejis, Mattila, and Schnur 1987) in the same position, but the ratio between $I_{\mathrm{v}}(100 \mu \mathrm{m})$ and $A_{\mathrm{v}}$ is about $1.7 \mathrm{MJy} \mathrm{sr}^{-1} \mathrm{mag}^{-1}$, which is much lower than the value $\left(18 \pm 3 \mathrm{MJy} \mathrm{sr}^{-1} \mathrm{mag}^{-1}\right)$ reported for typical cirrus clouds. This fact seems to indicate the presence of much cooler dust in the line of sight.

\section{REFERENCES}

de Lapparent, V., Geller, M., and Huchra, J. 1986, Ap. J. (Letters), 302, L1.

Hauser, M. G. et al. 1987, Ap. J. (Letters), 278, L15.

Laurejis, R. J., Mattila, K., and Schnur, G. 1987, Astron. Astrophys., 184, 269.

Murawski, W. 1983, Acta Cosmol., 12, 7.

Okroy, R. 1965, Astron. Cirk., 320, 4.

Rudnicki, K. and Wszolek, B. 1986, in Gamow Cosmology, Proc. of the int. school of physics, E. Fermi Course LXXXVI, eds. F. Melchiorri and R. Ruffini, North Holland. 